\title{
Feasibility of Advanced Vehicle Control Systems (AVCS) for Transit Buses
}

\author{
Robert A. Larsen
}

Raytheon-E Systems

\begin{abstract}
In the course of developing automated vehicle-roadway systems, opportunities to deploy vehicle control systems at intermediate stages of development may emerge. Some of these systems may provide a significant efficiency or safety enhancement to existing operations with manually-driven vehicles. Under certain circumstances, transit buses provide an ideal testbed for such systems. The work presented here represents a feasibility study for the application of Advanced Vehicle Control Systems (AVCS) to transit bus operations. The paper explores past and present research relevant to automatic control for buses and describes specific operations that could be better performed by AVCS-assisted or controlled vehicles.

The study concludes with a series of recommendations for proceeding toward a deployment phase. For transit bus operations, the most suitable deployment opportunities for AVCS exist on exclusive busways (bus-only roads) or large bus servicing facilities used for daily maintenance operations. Busways would provide an excellent testing ground for a lateral control/lane keeping system. Such a system would provide immediate utility on the existing busway and would serve as a building block for more highly automated systems in the future. Maintenance operations in service garages require dedicated drivers to move vehicles through a routine servicing sequence. By fully automating the movement of buses within such facilities, labor costs could be dramatically reduced.
\end{abstract}




\section{AVCS in Transit Buses-A Background}

While vehicle control has been extensively developed for rail/guidewaybased vehicles like trains and Automated People Movers (APMs), relatively little automation technology has been applied to buses. Likewise, despite underlying similarities among buses, automobiles, and trucks, the significant work performed in vehicle control for passenger cars (and to a lesser degree trucks) has largely gone untested for buses. On the one hand, this is surprising, given the sensitivity of transit operators to incremental improvements in operating efficiency-improvements that appear achievable through the application of AVCS. On the other hand, there is typically little funding available for the development of new transit technology, with available funds more likely spent on low-risk systems that show a more immediate return on investment. In addition to concerns regarding the cost-effectiveness of AVCS, there are many legal and institutional questions surrounding AVCS and vehicle automation-for example, liability issues in the case of accidents, as well as passenger and driver fears associated with the replacement of drivers by computers.

There is, however, a small body of work in transit bus guidance that demonstrates some of the potential benefits to be derived from AVCS. The most significant work has been demonstrated by the O-Bahn system, deployed in Adelaide, Australia; Essen, Germany; and the United Kingdom. The system provides automatic lateral control on express segments of the bus route and conventional (manual) vehicle control elsewhere. Special bus and roadway modifications are required for automatic operations. Both mechanically- and electronically-guided systems have been deployed since the late 1970s; however, the mechanicallyguided systems are much more commonly found in service. The mechanical system is guided by horizontal rollers connected to the steering linkage and projected from the sides of the bus, bearing against tall curbs. The electronicallyguided bus follows a current-carrying wire in the pavement using an inductive guidance principle. Similar in principle to conventional bus operations on exclusive bus lanes, the O-Bahn buses run on uncongested busways when under automatic control and on the conventional street network when under manual con- 
trol, providing benefits of rapid transit performance on line-haul segments and flexible collection/distribution service elsewhere. Furthermore, since the guided buses deviate only slightly from their busway lane, only a very narrow right-ofway is required. This allows for lower infrastructure costs and the ability to construct busways where very little space is available (particularly valuable for bridge and tunnel applications). As a result, O-Bahn systems may be viewed as a favorable alternative to light rail in some transit corridors. The ability to run in narrow rights-of-way may also allow guided buses to share subway rights-of-way with trains. This capability was demonstrated in Essen, allowing improved bus service in the downtown area by taking the buses off the congested surface streets and running them in underutilized rail tunnels.

In parallel with the work in guided buses has been the development of $\mathrm{Au}$ tomated Guideway Transit (AGT) systems. While these systems have been demonstrated using a wide range of vehicle and guideway designs significantly different than those used for bus systems, AGT's set a precedent for unmanned, fully autonomous transit vehicle control. Some notable examples of such systems have been deployed at airports around the world (Denver, Orlando, Chicago, etc.). Similar systems have been deployed in cities such as in Detroit, Miami, Lille (France), Vancouver, and London.

Personal Rapid Transit (PRT) concepts involving the use of small, automated guideway-based vehicles serving a dense network of origins and destinations have been investigated for at least 30 years, but the last few years have shown a renewed interest in these concepts as traffic congestion has worsened and technology has improved. Raytheon Electronic Systems of Marlborough, Massachusetts, is currently building a small PRT system for Northeastern Illinois Regional Transportation Authority (RTA) in Rosemont, Illinois, and feasibility studies of other systems are under way around the world. As an automated public transportation system, there are parallels between PRT and AHS (automated highway system) transit, but unlike mass transit, PRT attempts to provide automobile-like service, with very small vehicle capacities and point-to-point service. 


\section{Benefits of AVCS for Transit Buses}

In assessing the benefits of AVCS for transit buses, a review of existing transit bus operations was performed. From literature reviews, system tours, and interviews with transit experts, several operational areas emerged as suitable for AVCS improvement:

- lane keeping

- longitudinal control

- curbside docking

- maintenance operations

- collision avoidance

Each of these operational areas and the associated AVCS benefits are discussed below.

\section{Lane Keeping}

The performance of the lane-keeping task, common to all roadway vehicle operations, is more critical for wide vehicles like buses and trucks than for automobiles since lateral distances to the lane edges are reduced. Lane-keeping systems have been prototyped to provide various degrees of lane-centering control, ranging from driver warnings to full steering control. The value of a lane-keeping system exists for all road-going vehicles, particularly as an aid to driver inattention where lane changing is infrequent, such as freeway driving. However, there exist specific operations for transit buses that could be substantially improved with the aid of a lane-keeping system.

One example is operations in tunnels or other narrow segments of the bus right-of-way. Operations on these narrow segments require drivers to trade-off operating speed for safety. Our research found a substantial number of major transit bus operations with one or more narrow segments where buses must reduce speed or stop to ensure safety; a lane-keeping system does not need to be continuously engaged to provide benefits. A fatal 1996 head-on collision between two buses on a Pittsburgh busway can attest to the importance of the lanekeeping function. 
Other benefits of a lane-keeping system could accrue as the transit system infrastructure adapted to take full advantage of the bus's lateral control capabilities. Land acquisition and construction costs would be reduced where guided busways or segments are built as a result of reduced lane-width requirements. This advantage for laterally-guided buses would be most significant where adding or reallocating bridge or tunnel right-of-way is necessary.

\section{Longitudinal Control}

Operations that would benefit from the application of longitudinal control may take one of two forms: general automatic speed control or the special case of platooning. General automatic speed control would be employed to maintain desired headways between buses precisely for high frequency service (greater than 30 buses/hour) where slight headway variations could severely disrupt operations. Platooning represents the high frequency operational limit of speed control where headways approach several seconds or less. Longitudinal control systems employ sensors, typically vehicle-based, to control automatically the throttle and/or brakes, and, thus, vehicle speed. In the special case of platooning, a forward-looking radar, ladar, or other sensor, would be mounted on a bus to determine distance and closing rate with respect to the bus immediately ahead. The efficiency advantages of platooning vehicles are clearly demonstrated by the superior productivity of trains relative to buses on high-passenger-demand routes.

Perhaps the only U.S. operation of sufficient scale to justify platooning operates on the Lincoln Tunnel exclusive bus lane connecting northern New Jersey and Manhattan. Assuming available capacity in the Port Authority Terminal for additional incoming bus volumes, there exists the potential to expand the capacity of the bus lane further by applying longitudinal control systems to maintain very short headways safely between buses and keep the bus flow steady.

In the long term, a successful demonstration of platooning on an express lane might motivate transit planners to consider dedicated guided busways with bus platoons as an alternative to light rail in more heavily traveled corridors. 
This system conceivably could be demonstrated to run trains of buses under lateral and longitudinal control with a single lead driver (or perhaps no driver), to reduce labor costs significantly. Such a system could approach the operating efficiency of trains on moderately high-volume routes while utilizing much cheaper vehicles with the flexibility to be run on conventional roads. Autonomous vehicle-following technology has been demonstrated successfully for several years by various research institutes and vehicle manufacturers, including Daimler Benz, Carnegie Mellon University, and the National Institute of Standards and Technology (NIST), among others.

While the Lincoln Tunnel case would provide an opportunity to demonstrate longitudinal control to improve the capacity of an express segment of a bus route, much shorter platoons also could provide capacity benefits for non-express operations. The concept of a "virtual artic" (two or three platooned buses that move as a single bus with the passenger-carrying capacity of a single or double articulated bus) comes to mind. On some routes or route segments, it may be advantageous to utilize the operational efficiency of large capacity vehicles, even if each vehicle still retains a driver onboard.

Short of automatic platooning, a speed control system to precisely maintain short headways of approximately one minute or less would be advantageous on some high-volume transit lines. This approach could help to reduce the problem of bus bunching that often occurs on such routes when one bus slips from its schedule and following buses "close the gap" from behind.

\section{Curbside Docking}

The presence of a gap or height differential between bus doors and the curb/ platform area causes inefficient and inconvenient operations at bus stops. The provision of a level loading surface without gaps allows for much easier passenger access/egress and thus minimizes dwell time at stops. Another significant advantage for level loading is the improved access for the physically disabled. Level loading buses also eliminate the need for wheelchair lifts, which are expensive, maintenance intensive, and time-consuming to operate. In order to capture the advantages of level loading, however, there must be little or no gap 
between the bus and the curb, and, thus, automatic control of the bus for precise placement is desirable to ensure consistent and efficient docking. Over the past 20 years Volvo, and more recently, Renault have experimented with automatic bus guidance for this purpose.

\section{Maintenance Operations}

From discussions with several transit system operators, it is clear that any incremental reductions in operating expenses would be embraced. A significant number of operators interviewed believe that bus service and maintenance operations could be streamlined with the application of AVCS. Every day, there are routine operations repeated by dedicated maintenance staff who drive buses between stations to perform various tasks. At the end of each bus's service period, the driver takes the bus through a fueling area, a fluids check area, and a washing area, and then parks the bus in a designated space. An alternative to using drivers at each facility would be to move buses autonomously through the facility, either under their own power or by automated tow vehicles (see Figure 1). The relatively controlled environment of the maintenance area combined with the imme-

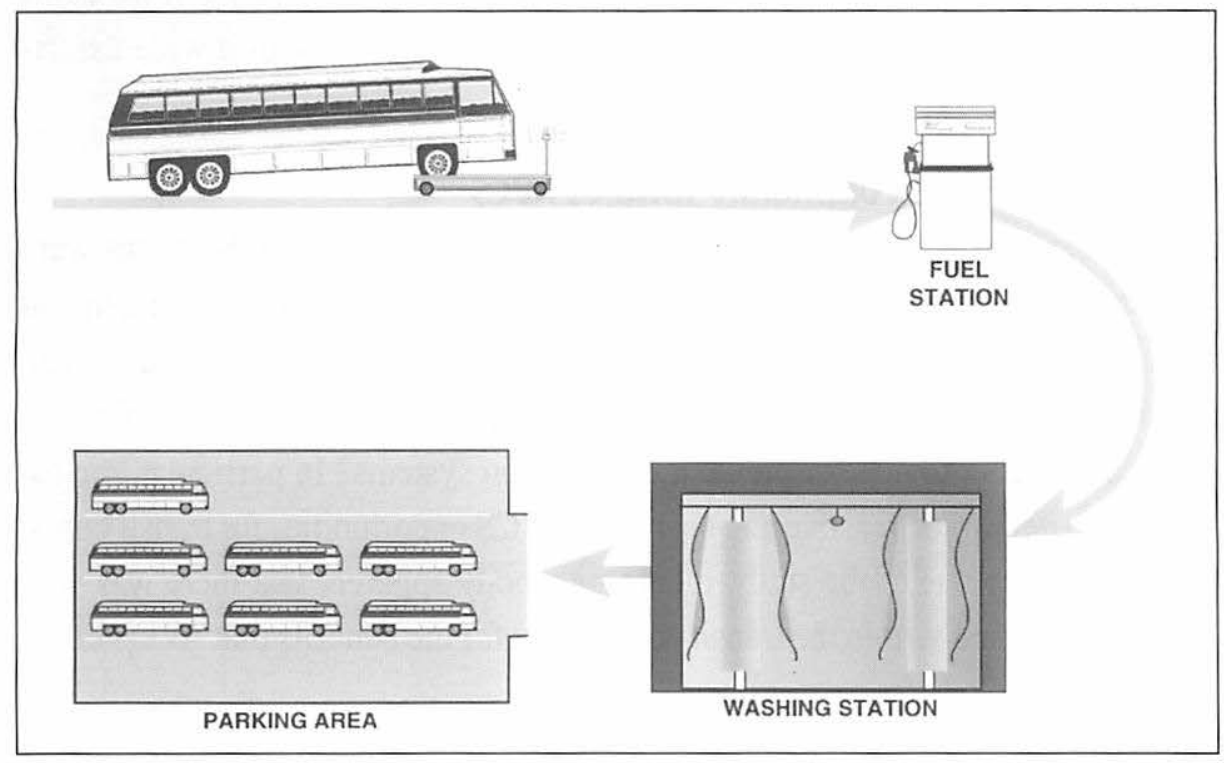

Figure 1. Concept for an automated bus servicing operation. 
diate benefits provided by AVCS make this a strong candidate for a system deployment.

The use of driverless tow vehicles, similar to those Automated Guided Vehicles (AGVs) that circulate in factories and warehouses around the world, could provide a direct replacement for maintenance facility drivers. In this scenario, each vehicle would be responsible for moving several buses per hour during servicing periods. Fully-automated buses would not require any dedicated drivers or tow vehicles, but a substantial fraction of the bus fleet would need to be equipped for automated movement to allow for significant operating cost savings.

\section{Collision Avoidance}

Like lane keeping, collision avoidance is under investigation for all types of vehicles. Several transit operators interviewed expressed interest in cost-effective collision-avoidance systems, particularly rear-end collision-avoidance systems. The National Highway Traffic Safety Administration (NHTSA) and various automotive manufacturers and suppliers are working actively toward collision avoidance systems to reduce the frequency and severity of a wide assortment of collision types.

\section{Attitudes of Transit Community Towards AVCS}

In the course of this research effort, many transit and AVCS studies were analyzed, and various transit industry experts were interviewed, including transit system operators, transit planners, bus manufacturers, transit consultants, and researchers. The question underlying this examination was: What tangible benefits can AVCS provide for public transportation systems? In particular, the focus was to determine feasible and near-term AVCS opportunities for transit buses. Through the course of the study, it became readily apparent that there was very little appreciation within the transit community for the benefits that AVCS could provide. 
Once the AVCS concept was thoroughly explained, the overall consensus of the transit community was that AVCS showed exciting potential for the distant future, but much less promise for the immediate future. The more visionary planners imagined dramatic service and operating cost improvements with guided buses running on busways and subway tracks and automated buses moving assembly line-style through maintenance garages, while less optimistic planners did not believe that AVCS could provide many significant benefits, even if the technological and institutional hurdles could be overcome. New technology comes slowly to the transit world, and vehicle control systems are perceived to be several steps beyond the current cutting-edge systems, which are typically information-flow-oriented, like real-time fleet management and traveler information systems. Transit managers cannot afford to be adventurous, either from a cost or operations standpoint, because there is little or no funding available for experimentation, and a system failure is unacceptable to the riders who rely on the service.

Most planners expressed concern that completely unmanned bus concepts would be difficult from a fare-collection and passenger security issue; however, they accepted that these concerns might possibly be addressed, at least in the short term, by providing lower-paid bus attendants on automated buses. Another concern expressed was that automation methods could displace drivers and upset labor relations. While many transit systems demonstrated opportunities for short- and long-term AVCS deployment, it is the long-term deployments (with facilities and vehicles designed to accommodate AVCS) that offer the highest payoffs. Unfortunately, the enabling technologies for the future must evolve from the short-term applications, like lane-keeping and other systems, which may not provide such a high cost-benefit advantage. Even the most pro-technology transit property will require a compelling economic analysis of the costs and benefits of an unproven technology approach like AVCS.

From the industry side, there was also cautious interest in AVCS. A leading transit industry consultant with expertise in the design and deployment of automated guided transit (AGTs) pointed out that with labor typically representing 
75 percent of operating costs, any incremental labor cost reduction that AVCS could provide should be considered seriously. He also indicated that it would be important to get the bus manufacturing industry interested in AVCS, as they would obviously need to contribute to the design and production of an AVCSequipped bus. This may be a challenge because the level of research and development funding is typically very low in the bus industry, and manufacturers would need to see a strong demand from their customers to justify any exploration of AVCS. Several European bus manufacturers, however, have proven their interest in vehicle control technology by deploying guided buses and investing in guidance technology.

\section{Feasible AVCS Technologies for Transit Bus Applications}

While this section is not intended to provide an exhaustive or thorough description of all guidance systems available, it attempts to illustrate the most promising technologies for a near-term system deployment. While several distinct systems are described here as alternatives, it is quite likely that the ideal AVCS for a given task will incorporate more than one of these technologies simultaneously.

\section{Wire Guidance}

As described previously, the inductive guidance system demonstrated on O-Bahn buses has a long history in vehicle control. This guidance technique, developed more than 40 years ago and widely used in factory automation for automated guided vehicles (AGVs), relies upon vehicle-mounted inductive coils to sense the magnetic field induced by current in the wire. The measured field indicates the distance between the coils and the wire, and, thus, vehicle lateral offset can be implied. Among its technical advantages, wire guidance is robust, proven, and relatively simple. Among its disadvantages, wire guidance is infrastructure-intensive and inherently inflexible, as it requires the presence of a wire path to any location that a vehicle may need to reach. 


\section{Passive Magnetic Trails}

Like the guided-wire system, the underlying guidance principle of magnetic trails is to provide a path in the pavement for a vehicle to follow easily. Unlike guided wires, however, passive magnetic trails do not require power to provide a guidance signal. Two approaches are currently under investigation: discrete magnetic markers and continuous magnetic stripe. The California Partners for Advanced Transit and Highways (PATH) program based at the University of California, Berkeley has investigated the discrete markers method and has successfully demonstrated its capability for lane keeping. Magnetic roadtape research is underway in Minnesota by $3 \mathrm{M}$. Their work focuses on the incorporation of a magnetic substrate into a conventional pavement marking tape. Like wire-guided systems, magnetic trails may provide reliable and accurate lane keeping, but they are infrastructure-intensive and relatively inflexible.

\section{Differential Global Positioning System}

The Global Positioning System (GPS) has been used for several years in the tracking of vehicles, seacraft, aircraft, etc. The system that incorporates line-ofsight communications between orbiting satellites and a receiver anywhere on earth provides positional accuracy on the order of $100 \mathrm{~m}$ for general users. To greatly improve accuracy, signal processing enhancements, generally classified as differential GPS (DGPS), have been introduced to correct signal transmission degradation between the satellites and a receiver. Research in recent years has shown that DGPS can provide positional accuracy in the $2 \mathrm{~cm}$ range-sufficient to make this technology feasible as a navigation system. While there are disadvantages associated with GPS, its major inherent advantages are high accuracy and existing infrastructure availability (satellites and ground stations). Many in the AVCS community believe that, in the future, DGPS will provide one of the basic guidance technologies for vehicles.

\section{Machine Vision}

Image processing techniques have been under development for many years and have been successfully implemented in automobiles and other mobile robots for guidance. Among advantages, machine vision systems require little or no 
infrastructure modifications, have been shown to provide excellent positional data for vehicle guidance, and may be configured to perform many different tasks (from lane keeping to collision avoidance to road sign reading). Some disadvantages are current system expense, complexity, and inherent limitations of the basic sensor (camera), which can provide information only on the scene immediately visible to it.

\section{Opportunities in Specific Transit Systems}

Over the course of several months in late 1995 and early 1996, transit managers at several transit agencies were interviewed to assess their interest in AVCS for transit bus operations. The following is a summary of findings from those discussions.

\section{Pittsburgh}

Of transit properties studied, the Port Authority Transit (PAT) system is one of the most suitable for AVCS deployment. PAT operates the only dedicated and grade-separated busways in the country, providing an excellent testbed for vehicle control testing and development. Based on conversations with PAT staff, it appears that they are generally receptive to new technologies that can legitimately reduce operating costs or improve service quality.

\section{Houston}

With its well-funded and heavily bus-oriented transit system, Houston is currently the only regional transit agency spending research and development funds on the development of AVCS. Houston METRO is scheduled to participate in the 1997 AHS Demonstration with laterally- and longitudinally-guided buses based on machine vision and forward-looking radar sensors. They have also expressed serious interest in the testing of automated movement of buses within maintenance facilities.

\section{Cleveland}

The Greater Cleveland Regional Transit Authority (RTA) staff were interested in AVCS and in new transit technology in general; some were particularly fascinated by the potential of AVCS for RTA's operations. Of particular interest 
was the maintenance area automated vehicle concept previously described. Interest was also expressed by RTA planners for the Euclid Avenue corridor, which will undergo a major bus transit service improvement in the next several years. An option that may be considered for the corridor is a guided busway, given the very limited right-of-way available.

\section{Seattle}

Some features of King County Metro's transit system make it a suitable candidate for AVCS deployment. The unique 1.3-mile bus tunnel/subway and attached busway segment are exclusive bus facilities that show potential safety and efficiency benefits from AVCS. The automated service garage concept was also of interest to Metro planners. Furthermore, the director of King County Department of Transportation (KCDOT) is a strong proponent of new technology for his transit system.

\section{Other Areas}

In addition to the specific cities listed above, there are other cities and regions that may also be suitable for an AVCS deployment. In the course of this study, it became clear that transit systems in each city have their own unique opportunities for AVCS, whether it be for narrow tunnel segments, dedicated bus lanes, abandoned or shared rail rights-of-way, or other opportunities. Some of the more promising transit AVCS opportunities exist in such areas as metropoli$\tan$ New York City, Minneapolis, and Montgomery County, Maryland. An interesting development that may encourage the introduction of AVCS is the increasing popularity of busways. While very few dedicated busways exist in the U.S. today, many transit planners are now considering busways and occasionally guided busways as alternatives in their corridor studies (Boston, Milwaukee, and Raleigh are examples). These bus-only facilities are the most suitable for the adaptation of lateral and longitudinal control systems, as they present a relatively controlled environment for integrating new equipment on buses and the facility itself. 


\section{Recommendations for Future Work}

From a review of transit industry needs and available AVCS technologies, some recommendations have been identified for continued work in the near term:

Automation of bus movement through service areas in bus garages was the most popular AVCS vision for transit operators. Some managers asked how much a system of this type would cost. This should be a high priority area of study for future work. Specifically, a detailed study of vehicles, facilities, and servicing operations at an interested transit property should be performed, and a small handful of AVCS technology providers should be contacted to work toward developing alternative design concepts and cost estimates for such a system.

A design concept and cost estimate for a lateral control system for lane keeping should be developed. As described previously, there are many potential benefits for lane-keeping systems in the near and long terms as well as many levels of deployment possible, from warning systems to full lateral control. In cooperation with specific technology providers, transit agencies, and bus manufacturers, alternative system concepts should be developed and a cost estimate established for each deployment alternative.

With regard to the second option, successful deployment of a lane-keeping system requires that the system perform as designed and be accepted by the transit industry as a legitimate operational enhancement for buses. To achieve this goal, two parallel paths should be taken to enhance the likelihood of success. The first path should focus on a limited deployment of a system for revenue service operations. It is clear from discussions with transit operators that serious consideration of new technology will follow only from real-world demonstrations. It is proposed that a deployment plan include a single, laterally-guided bus operating passenger service on an existing route/roadway segment. This would provide a relatively low-cost technical feasibility demonstration with real credibility for transit operators.

At the same time, efforts should be made to demonstrate the economic justification for a lane-keeping system. A guided-busway alternative based on modern AVCS technology (lane keeping) could prove superior to typical transit 
alternatives like conventional busways and rail systems. The reduced right-ofway advantage of a guided busway relative to a conventional busway is highly significant in some travel corridors. This advantage needs to be quantified in economic terms; a thorough analysis of the costs and benefits associated with a guided busway alternative relative to conventional alternatives should be developed for a suitable transit corridor.

Once transit operators are convinced of the technical feasibility and economic justification for lane-keeping systems, it should only be a matter of time before deployment begins.

\section{Conclusion}

Through the course of this study, numerous contacts within the transit industry were interviewed, and four major transit operations were toured and reviewed. While tremendous opportunity exists for AVCS in transit, successful implementation will require cautious steps. Short-term benefits of AVCS certainly can be demonstrated with modifications to existing vehicles and infrastructure, but to capture fully the larger, long-term benefits will require that vehicles, infrastructure, AVCS equipment, and many transit agency processes (like route planning, scheduling, and operations) be coordinated as a unified system. In the course of this study, two significant observations have emerged:

- Very little shared knowledge exists between the AVCS and transit communities.

- Like so many other pioneering intelligent transportation systems (ITS) initiatives, the deployment of AVCS for public transit will encounter more significant institutional and legal hurdles than technical challenges.

The importance of the first point cannot be overstated. Effective system design requires understanding the entire system and the interactions between all the components. From a technical standpoint, an effective, large-scale AVCS deployment would require a detailed understanding of issues associated with bus operations, vehicles, infrastructure, sensor technology, control system design, and many other issues. The second point indicates the importance of incorporat- 
ing many non-technical issues into the design process. There are major financial considerations, as well as legal and institutional barriers. There are transit system managers, transit employees, and the riding public who would all need to accept the changes that AVCS would bring. From the standpoint of the transit management there are many risks associated with AVCS, not the least of which are angry labor unions and law suits in the case of system failure. With so little funding available for new technology at most agencies, there is a high opportunity cost associated with testing relatively unproven technology.

If automated highways and automated transit are to achieve broad public acceptance, the transit bus offers an excellent platform for initial deployment. The basic vehicle and infrastructure already exist, and incremental AVCS deployments like lane-keeping systems can demonstrate real benefits while limiting financial, legal, and institutional risks associated with more extensive deployment scenarios. Ultimately, the evolution of vehicle control systems for buses promises to raise the general level of acceptance of automation technology and allow for the increased mobility, safety, and efficiency that automation provides.

\section{Acknowledgments}

The author wishes to thank Dick Bishop and Nick Panebianco of the Federal Highway Administration for their support of this study. Thanks also to Ron Fisher of Creative Transit Alternatives for his substantial input and guidance throughout the program.

\section{About the Author}

ROBERT LARSEN is a transportation engineer with Raytheon E-Systems in Falls Church, Virginia, and the principal investigator on an FHWA-sponsored study of advanced vehicle control systems (AVCS). Mr. Larsen holds a bachelor's degree in engineering sciences from Harvard and a master's degree in transportation engineering from the University of Maryland. 